Article

\title{
High Aspartate Aminotransferase/Alanine Aminotransferase Ratio is the Conventional Biochemical Biomarker that is Most Closely Associated with All-Cause Mortality in Older People
}

\author{
Kei Nakajima ${ }^{1, *}$, Mariko Yuno, Kazumi Tanaka and Teiji Nakamura \\ 1 School of Nutrition and Dietetics, Faculty of Health and Social Services, Kanagawa University of Human \\ Services, 1-10-1 Heisei-cho, Yokosuka, Kanagawa 238-8522, Japan \\ 2 Department of Endocrinology and Diabetes, Saitama Medical Center, Saitama Medical University, 1981 \\ Kamoda, Kawagoe, Saitama 350-8550, Japan \\ * Correspondence: nakajima-rsh@kuhs.ac.jp; Tel: +81-046-828-2660; Fax: +81-046-828-2661
}

\begin{abstract}
Low serum alanine aminotransferase activity and high aspartate aminotransferase (AST)/ALT ratio may be associated with high mortality in older people. We aimed to confirm this in an 8-year retrospective cohort study. Clinical data for 5,958 people aged 67-104 years were analyzed for their relationships with all-cause mortality using artificial intelligence (AI; Prediction One [Sony Network Communications Inc.]) and conventional statistical analysis (SAS Enterprise Guide [SAS Institute Inc.]). In total, 1,413 (23.7\%) participants died during the study. Auto-AI analysis with five rounds of cross-validation showed that AST/ALT ratio was the third largest contributor to mortality, following age and sex. Serum albumin concentration and body mass index were the fourth and fifth largest contributors, and the individual serum ALT and AST activities were the seventh and tenth largest contributors. Conventional survival analysis showed that ALT, AST, and AST/ALT ratio as continuous variables were all associated with mortality (adjusted hazard ratios (95\% confidence intervals): 0.98 (0.97-0.99), 1.02 (1.02-1.03), 1.46 (1.32-1.62), respectively; all $p<0.0001)$. In conclusion, both AI and conventional analysis suggest that of the conventional biochemical markers, high AST/ALT ratio is most closely associated with all-cause mortality in older people.
\end{abstract}

Keywords: alanine aminotransferase; aspartate aminotransferase; AST/ALT ratio; mortality; artificial intelligence; older people

\section{Introduction}

It can be difficult to identify older members of the community who are at a high risk of mortality, particularly using the conventional biochemical parameters measured in clinical practice. During the past two decades, many studies have shown that low serum alanine aminotransferase (ALT) activity and high aspartate transaminase (AST)/ALT ratio are associated with high mortality in older people [1-13]. However, the mechanisms involved remain to be conclusively established [14-17]. Some previous studies have also shown that high AST activity is associated with mortality $[18,19]$. In addition, we previously showed that high serum AST activity, accompanied by normal serum ALT activity, is associated with underweight owing to weight loss [20], which are both risks for mortality.

In clinical practice, the circulating activities of ALT and AST are routinely measured to identify hepatic disease [21,22]. Both transaminases have metabolic functions, in particular in amino acid metabolism, and are expressed in multiple organs, including the liver, myocardium, and skeletal muscle $[23,24]$. Specifically, ALT is primarily expressed in the liver, whereas AST is expressed more widely, including in skeletal muscle. Therefore, a high serum ALT activity reflects the destruction of hepatocytes, whereas a high serum AST activity, alongside a normal ALT activity, may reflect muscle damage. In 
younger and middle-aged people, long-term increases in serum ALT activity are caused for example by non-alcoholic fatty liver disease [25,26], but eventually the hepatic transaminase activities of the patients may become low during the terminal stage of cirrhosis because of lower ALT synthesis in the liver. In contrast, in older people, a low serum ALT activity might be caused by age-related atrophy of the liver or other organs, including the muscles and lungs $[27,28]$. Given these variations in serum ALT and AST activity, the AST/ALT ratio may more accurately reflect the risk of mortality. Therefore, we performed an 8-year community-based retrospective cohort study, in which we used both artificial intelligence (AI) and conventional statistical analysis to investigate the relationships of this ratio and other biochemical markers with the risk of mortality. AI techniques such as machine learning and deep learning have been shown to improve the prediction of diseases, such as cardiovascular disease [29].

\section{Materials and Methods}

\subsection{Study design}

We performed an 8-year cohort study of healthcare data relating to people living in Yamato City in Kanagawa Prefecture. After the conclusion of a contract between Yamato City and Kanagawa University of Human Services, we received the data, which comprised clinical parameters, nursing care level, and mortality, but not the cause of death. The study protocol was approved by the ethics committee of Kanagawa University of Human Services (Approval number 17-26) and the study conformed with the principles of the Declaration of Helsinki. The requirement for informed consent was waived because it was a retrospective study of anonymized data. The study protocol was available on the home page of the university [30] and was published in the public relations magazine of Yamato City [31].

\subsection{Participants and measurements}

Data collected for 6,068 people living in Yamato City, Kanagawa Prefecture, who were aged $\geq 64$ years and underwent a baseline examination between April 2011 and March 2012, were reviewed. Owing to incomplete or missing data, 110 individuals were excluded, leaving 5,958 for the analysis. Specific inclusion and exclusion criteria were not used, but all the participants had undergone an annual check-up and responded to questions prepared by the medical personnel, which implies that they were not at imminent risk of death, nor did they have severe dementia at baseline.

Clinical measurements were made in the morning after an overnight fast. Body mass index (BMI) was calculated as body mass $(\mathrm{kg})$ divided by the square of height $\left(\mathrm{m}^{2}\right)$. Serum biochemical parameters were measured automatically using standard methods. Estimated glomerular filtration rate (eGFR) was calculated using the equation developed for Japanese populations [32]:

$\operatorname{eGFR}\left(\mathrm{ml} / \mathrm{min} / 1.73 \mathrm{~m}^{2}\right)=194 \times \mathrm{Cr}^{-1.094} \times \operatorname{Age}^{-0.287}(\times 0.739$ if female $)$, where $\mathrm{Cr}$ is the serum creatinine concentration $(\mathrm{mg} / \mathrm{dL})$.

Questions regarding lifestyle, including smoking habits, alcohol consumption, and pharmacotherapy for hypertension, diabetes, and dyslipidemia, were developed by the municipal officers of Yamato City, referring to the questions prepared by the Japanese Ministry of Health, Labour and Welfare in 2008 for use in health check-ups in Japan.

\subsection{Auto-AI analysis}

We performed a graphical user interface (GUI) based automatic AI (auto-AI) analysis using Prediction One (Sony Network Communications Inc., Tokyo, Japan) [33] and appropriately prepared datasheets. This auto-AI analysis system does not require specific user skills. Data learning, evaluation, and neural network analysis were automatically performed during the analysis to generate an optimal prediction model, with internal cross-validation. The output was a list of parameters, ranked according to the level of contribution of each. 
Unfortunately, as previously described [34], the details of the AI, including the neural network architecture, are trade secrets and therefore not available for publication.

The contributors to mortality were listed in order, according to their contribution (weight). The weight of contributor, which is considered as "gain of feature importance", is equivalent to the relative contribution calculated using machine learning algorithms such as extreme gradient boosting (XGBoost) and logistic regression [35].

For each parameter, the incidences of positive and negative outcomes are shown. After many trials had been conducted using the auto-AI analysis, parameters with weightings $<0.003$ were excluded from the contributors. Ten factors (age; sex; smoking status; frequency of alcohol consumption; pharmacotherapy for hypertension, diabetes, and dyslipidemia [yes or no]; BMI; white blood cell count; serum albumin concentration; eGFR; the serum AST, ALT, and gamma-glutamyltransferase [GGT] activities; and the AST/ALT ratio) remained as explanatory factors for all-cause mortality.

In the AI analysis, 259 participants whose survival could not be confirmed after 8 years, because they had moved or for other reasons, were excluded. The area under the curve (AUC) for each predictive model was calculated, with $>74 \%$ and $63 \%-73 \%$ being considered to indicate a good and a standard predictive model, respectively [33].

\subsection{Conventional statistical analysis}

Data are expressed as mean \pm standard deviation (SD) or median (interquartile range). We used the SAS Enterprise Guide (SAS-EG 7.1) in SAS software, version 9.4 (SAS Institute, Cary, NC, USA), which has been used for numerous medical studies worldwide for several decades. Although SAS software also involves an AI system, the procedures involved are complex, particularly for non-SAS users, and require some technical skills, unlike in the case of the auto-AI described above.

The Kaplan-Meier method was used for all-cause mortality analysis. The Log rank and Wilcoxon Tests were used to compare participants categorized according to the quartiles of baseline AST/ALT ratio and serum ALT activity. A Cox proportional hazard model, in which the time elapsed until death was considered, was used to calculate the adjusted hazard ratios (HRs) associated with clinical parameters (serum ALT, AST, and AST/ALT ratio; continuous variables). Conventional statistical analyses were performed using SAS Enterprise Guide (SAS-EG 7.1) in the SAS system, version 9.4 (SAS Institute, Cary, NC, USA). $p<0.05$ was considered to represent statistical significance

\section{Results}

The baseline characteristics of the participants are shown in Table 1. Although the mean systolic blood pressure of the participants was higher than that of the general Japanese population, the mean values of the other parameters were almost normal. Death occurred in 1,413 $(23.7 \%)$ participants during the 8 years of the study. In addition, a further $1,632(27.4 \%)$ participants developed overt disability (long-term care level $\geq 2$ ) during the same period.

Table 1. Baseline clinical characteristics of the participants.

\begin{tabular}{cc}
\hline $\mathbf{N}$ & $\mathbf{5 , 9 5 8}$ \\
\hline Men, $\mathrm{n}(\%)$ & $3,430(57.6)$ \\
Age (years) & $79.4 \pm 3.9$ \\
Range & $67-104$ \\
Body mass index $\left(\mathrm{kg} / \mathrm{m}^{2}\right)$ & $22.7 \pm 3.2$ \\
Systolic blood pressure $(\mathrm{mmHg})$ & $132 \pm 16.0$ \\
Blood parameters & \\
Alanine aminotransferase (ALT) (IU/L) & $16(13-21)$ \\
Aspartate aminotransferase (AST) (IU/L) & $22(19-26)$ \\
\hline
\end{tabular}




\begin{tabular}{cc}
\hline$\gamma$-glutamyl transferase (IU/L) & $20(15-30)$ \\
AST/ALT ratio & $1.38(1.15-1.64)$ \\
Triglyceride $(\mathrm{mg} / \mathrm{dl})$ & $98(73-134)$ \\
HbA1c $(\%, \mathrm{NGSP})$ & $61 \pm 15.8$ \\
Albumin $(\mathrm{g} / \mathrm{mL})$ & $5.3 \pm 0.6$ \\
High-density lipoprotein -cholesterol $(\mathrm{mg} / \mathrm{dl})$ & $4.2 \pm 0.3$ \\
White blood cell $\left(\times 10^{3}, \mu / \mathrm{mL}\right)$ & $5.6 \pm 1.5$ \\
History of cardiovascular disease, $\mathrm{n}(\%)$ & $877(14.7)$ \\
Daily alcohol consumption, $\mathrm{n}(\%)$ & $1,129(19.0)$ \\
Current smoker, $\mathrm{n}(\%)$ & $390(6.6)$ \\
Having regular exercise, $\mathrm{n}(\%)$ & $3,185(53.5)$ \\
\hline
\end{tabular}

Table 2. shows the contributors to all-cause mortality. The AUCs for models 1 and 2 were $73.1 \%$ and $72.2 \%$, implying that these were good prediction models. 
Table 2. Parameters contributing to the prediction of all-cause mortality.

\begin{tabular}{|c|c|c|c|c|}
\hline \multirow{2}{*}{$\begin{array}{l}\text { Degree of contribution }- \\
\text { (Order) }\end{array}$} & \multicolumn{2}{|l|}{ Model 1} & \multicolumn{2}{|l|}{ Model 2} \\
\hline & Variables & Weight & Variables & Weight \\
\hline 1 & Age (years old) & 0.236 & Age (years old) & 0.229 \\
\hline 2 & Sex (men,1; women,2) & 0.119 & Sex (men,1; women,2) & 0.118 \\
\hline 3 & AST/ALT ratio & 0.094 & Body mass index $\left(\mathrm{kg} / \mathrm{m}^{2}\right)$ & 0.094 \\
\hline 4 & Serum albumin $(\mathrm{g} / \mathrm{dL})$ & 0.092 & Serum albumin $(\mathrm{g} / \mathrm{dL})$ & 0.094 \\
\hline 5 & Body mass index $\left(\mathrm{kg} / \mathrm{m}^{2}\right)$ & 0.089 & White blood cell $\left(\times 10^{3} / \mu \mathrm{L}\right)$ & 0.085 \\
\hline 6 & White blood cell $\left(\times 10^{3} / \mu \mathrm{L}\right)$ & 0.083 & eGFR (ml/min/1.73m²) & 0.073 \\
\hline 7 & eGFR (ml/min/1.73m²) & 0.076 & Serum ALT (U/dL) & 0.066 \\
\hline 8 & Serum GGT (U/dL) & 0.063 & Serum GGT (U/dL) & 0.056 \\
\hline 9 & Current smoking (yes; 1, no; 0 ) & 0.053 & Current smoking (yes; 1, no; 0 ) & 0.053 \\
\hline 10 & $\begin{array}{l}\text { Pharmacotherapy for hypertension (yes; } 1, \\
\text { no; } 0 \text { ) }\end{array}$ & 0.041 & Serum AST (U/dL) & 0.049 \\
\hline 11 & Pharmacotherapy for diabetes (yes; 1, no; 0 ) & 0.038 & $\begin{array}{l}\text { Pharmacotherapy for hypertension (yes; } 1 \text {, no; } \\
0 \text { ) }\end{array}$ & 0.040 \\
\hline 12 & $\begin{array}{l}\text { Pharmacotherapy for dyslipidemia (yes; } 1, \\
\text { no; } 0 \text { ) }\end{array}$ & 0.038 & $\begin{array}{l}\text { Pharmacotherapy for dyslipidemia (yes; } 1 \text {, no; } \\
\text { 0) }\end{array}$ & 0.034 \\
\hline 13 & Alcohol consumption & 0.031 & Alcohol consumption & 0.033 \\
\hline 14 & & & Pharmacotherapy for diabetes (yes; 1, no; 0 ) & 0.030 \\
\hline $\begin{array}{l}\text { Total classification } \\
\text { accuracy (AUC) }\end{array}$ & $73.1 \%$ & & $72.2 \%$ & \\
\hline
\end{tabular}

In Model 2, the AST and ALT activities were separately included as independent variables. Alcohol consumptions of "almost none", "sometimes", or "daily" were coded as 1, 2, and 3, respectively. ALT, alanine aminotransferase; AST, Aspartate aminotransferase; GGT, $\gamma$-glutamyl transferase. 
The AI analysis ranked high AST/ALT ratio as the third greatest contributor to mortality, following older age and male sex in Model 1. Serum albumin concentration and BMI were the fourth and fifth-ranked contributors to mortality. The individual serum ALT and AST activities were the seventh and tenth greatest contributors in Model 2.

Figure 1. shows the ranges and weightings of the AST/ALT ratio and ALT with respect to mortality. AST/ALT ratio $\geq 1.64$ (2), (3), (1)) was a positive contributor and AST/ALT ratio $\leq 1.50$ (2), (3), (1) ) was a negative contributor to mortality.
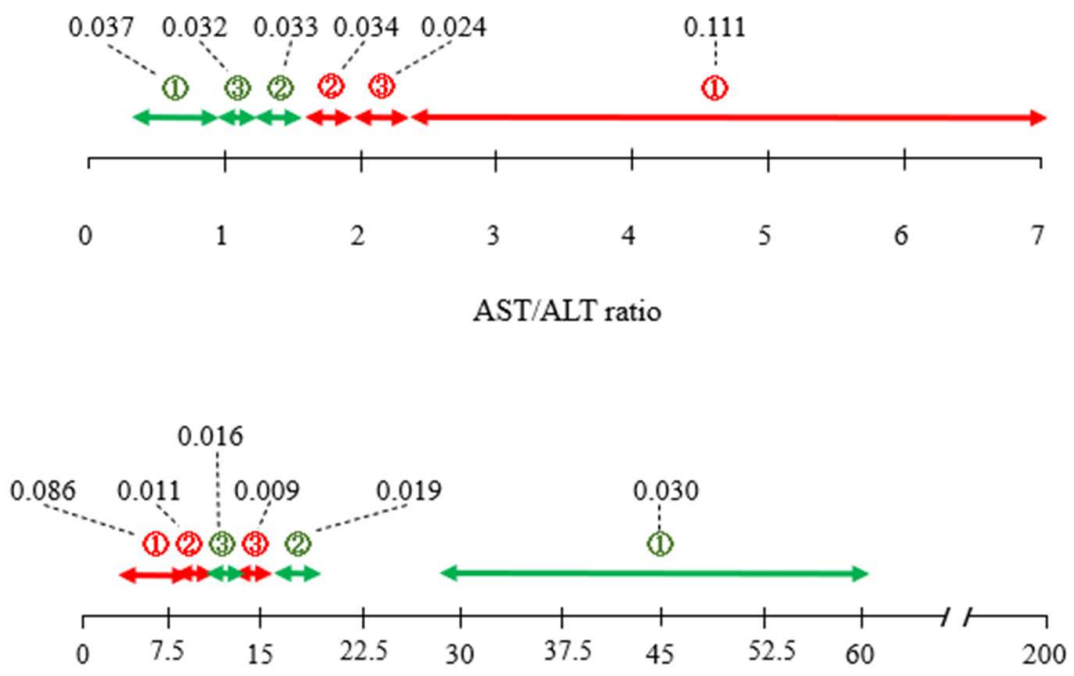

Serum ALT (U/L)

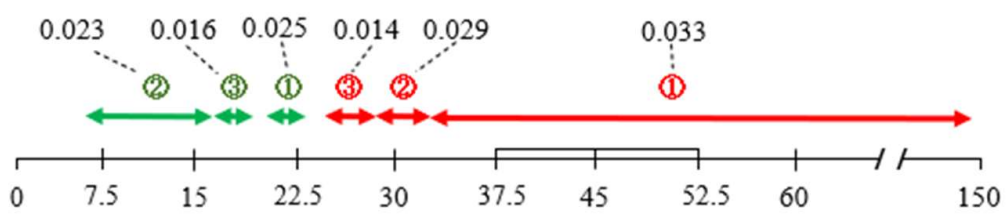

Serum AST (U/L)

Figure 1. Detailed contributions (ranges and weightings) of AST/ALT ratio, ALT activity, and AST activity to the risk of mortality, according to the AI analysis. Red and green arrows indicate positive and negative contribution to the risk of mortality. (1): First degree contribution. (2): Second degree contribution (3): Third degree contribution The space in the bar indicates the categories with lower contributions ( $\geq$ fourth degree). ALT; alanine aminotransferase, AST; aspartate transaminase.

When ALT and AST were considered individually, serum ALT activity $\leq 16 \mathrm{U} / \mathrm{L}$ (3), (2), (1)) was a positive contributor and serum ALT $\geq 12 \mathrm{U} / \mathrm{L}$ (3), (2), (1) was a negative contributor to mortality, indicating that the ALT ranges associated with each outcome overlap. Serum AST $\geq 25 \mathrm{U} / \mathrm{L}$ (3), (2), (1)) was a positive contributor and serum AST $\leq 23$ U/L (1), (3), (2) was a negative contributor. Overall, the contributions of the serum ALT and AST activities were smaller than that of AST/ALT ratio. Of all the ranges calculated, the weighting of 0.111 for an AST/ALT ratio of $\geq 2.30$ (1) was the largest and that of 0.086 for an ALT activity of $\leq 10$ (1) was the second largest.

Figure 2 and Figure 3 show the Kaplan-Meier survival curves for the mortality of the participants, which were categorized according to quartiles of AST/ALT ratio and serum ALT activity, respectively. Participants with a high AST/ALT ratio and a low ALT activity 
at baseline were at significantly higher risk of mortality during the study (Log rank and Wilcoxon Tests, both $p<0.0001)$. However, Figure 3 shows that after the lowest ALT activity ( $\leq 16 \mathrm{U} / \mathrm{L}, \mathrm{Q} 1)$, the highest ALT activity $(\geq 22 \mathrm{U} / \mathrm{L}, \mathrm{Q} 4)$ was also associated with high mortality.

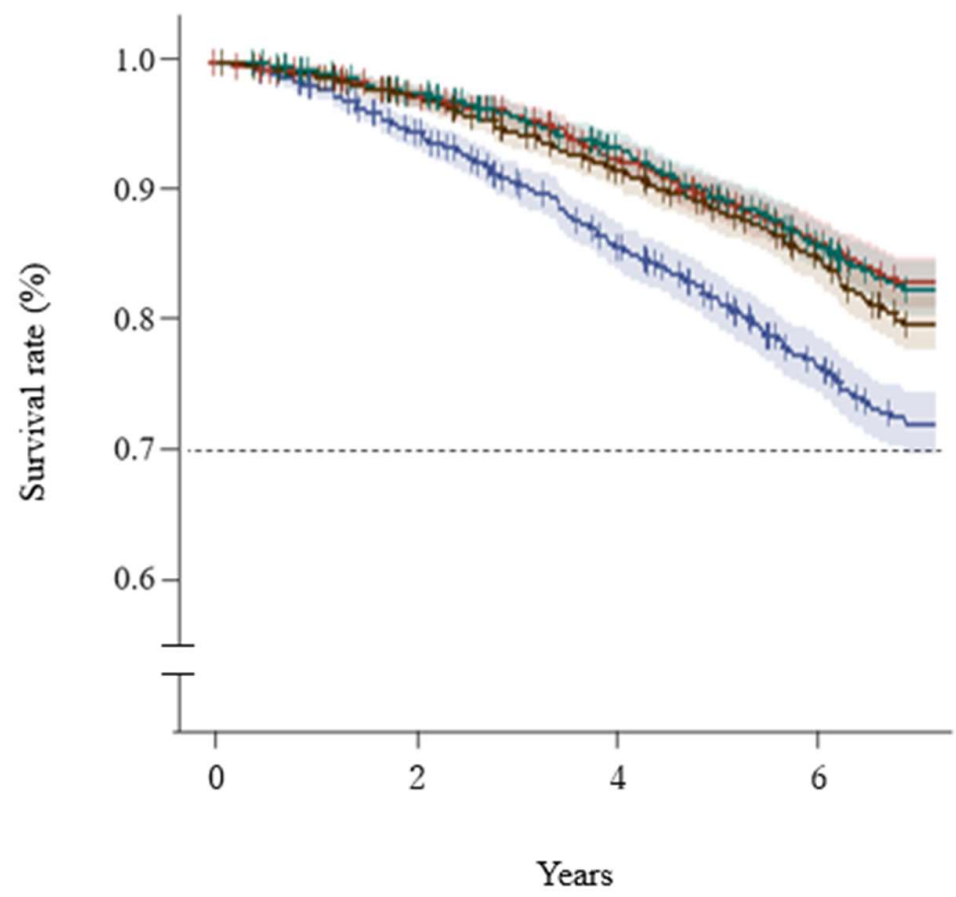

Figure 2. Survival of the participants, categorized according to quartile of AST/ALT ratio. Blue line, $\geq 1.64$ (Q1); brown line, 1.38-1.63 (Q2); green line, 1.15-1.37 (Q3); red line, $\leq 1.14$ (Q4). The colored areas surrounding the lines show the $95 \%$ confidence intervals. ALT; alanine aminotransferase, AST; aspartate transaminase. 


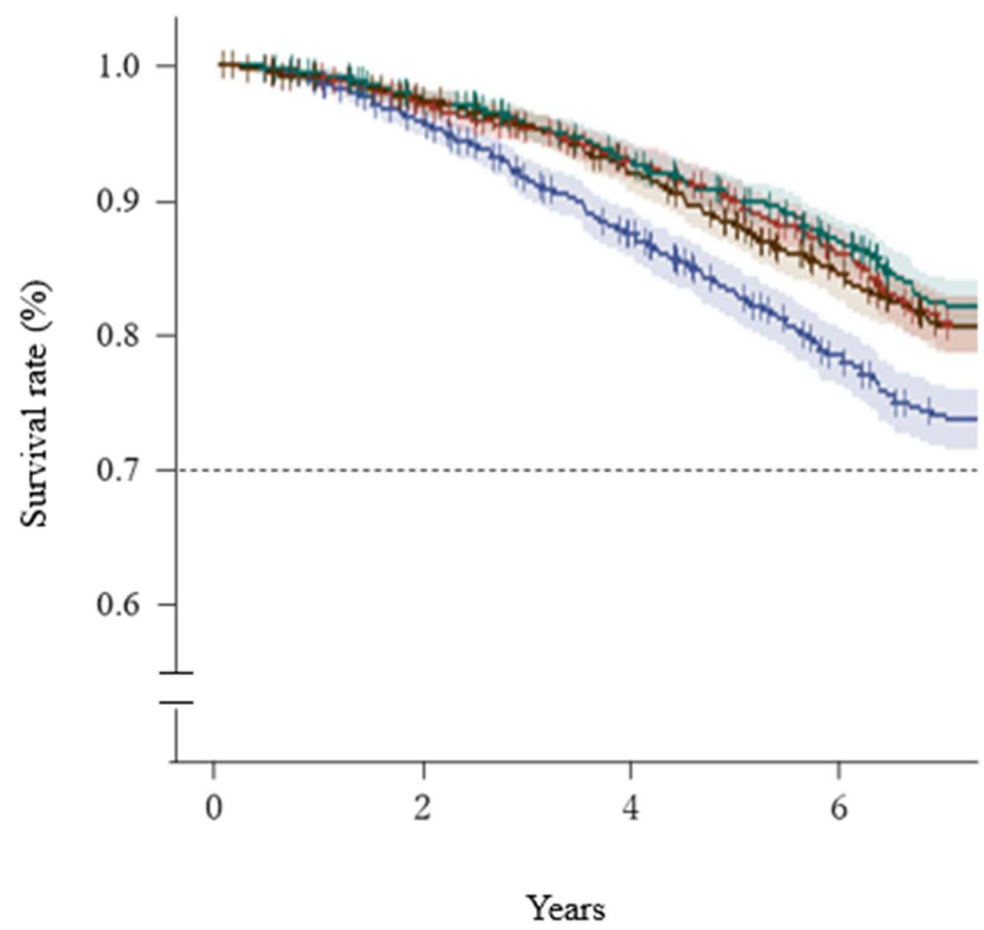

Figure 3. Survival of the participants, categorized according to quartile of serum ALT activity. Blue line, $\leq 12 \mathrm{U} / \mathrm{L}$ (Q1); red line, 13-16 U/L (Q2); green line, 17-21 U/L (Q3); brown, $\geq 22 \mathrm{U} / \mathrm{L}$ (Q4). The colored areas surrounding the lines show the $95 \%$ confidence intervals. ALT; alanine aminotransferase.

Cox proportional hazard analysis (Table 3) showed that AST/ALT ratio as a continuous variable was significantly positively associated with all-cause mortality in Model 1. In addition, serum ALT was significantly negatively associated and AST was significantly positively associated with all-cause mortality in Model 2. Each model was adjusted for the potential confounding factors listed in Table 2. Of note, eGFR was not associated with mortality, even though the AI analysis showed eGFR to be the sixth or seventh greatest contributor (Table 2).

Table 3. Adjusted hazard ratios for all-cause mortality associated with each parameter.

\begin{tabular}{|c|c|c|c|}
\hline & Variables & $\begin{array}{c}\text { Hazard ratios } \\
(95 \% \mathrm{CIs})\end{array}$ & $P$ value \\
\hline \multicolumn{4}{|l|}{ Model 1} \\
\hline & Age (years old) & $1.12(1.11-1.13)$ & $<.0001$ \\
\hline & Sex (men,1; women,2) & $0.48(0.42-0.54)$ & $<.0001$ \\
\hline & AST/ALT ratio & $1.46(1.32-1.62)$ & $<.0001$ \\
\hline & Serum albumin (g/dL) & $0.44(0.37-0.53)$ & $<.0001$ \\
\hline & Body mass index $\left(\mathrm{kg} / \mathrm{m}^{2}\right)$ & $0.96(0.94-0.98)$ & $<.0001$ \\
\hline & White blood cell $\left(\times 10^{3} / \mu \mathrm{L}\right)$ & $1.06(1.03-1.10)$ & 0.0001 \\
\hline & $\mathrm{eGFR}\left(\mathrm{ml} / \mathrm{min} / 1.73 \mathrm{~m}^{2}\right)$ & $1.00(0.99-1.00)$ & 0.250 \\
\hline & Serum GGT (U/dL) & $1.00(1.00-1.01)$ & $<.0001$ \\
\hline & Current smoking (yes; 1, no; 0 ) & $1.31(1.10-1.57)$ & 0.003 \\
\hline
\end{tabular}




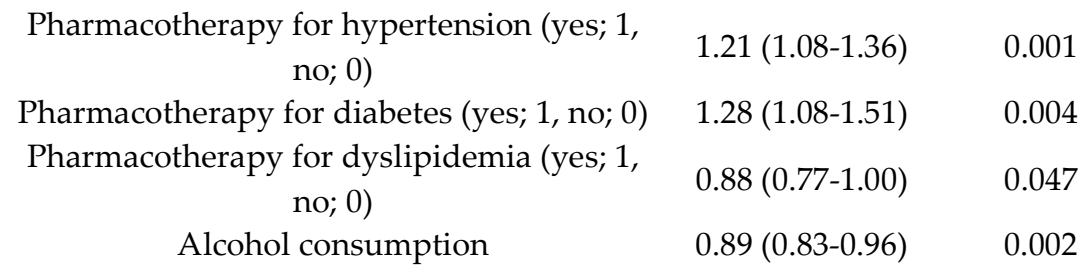

Model 2

\begin{tabular}{ccc} 
Age (years old) & $1.12(1.11-1.14)$ & $<.0001$ \\
Sex (men,1; women,2) & $0.49(0.43-0.55)$ & $<.0001$ \\
Body mass index $\left(\mathrm{kg} / \mathrm{m}^{2}\right)$ & $0.95(0.94-0.97)$ & $<.0001$ \\
Serum albumin $(\mathrm{g} / \mathrm{dL})$ & $0.44(0.36-0.53)$ & $<.0001$ \\
White blood cell $\left(\times 10^{3} / \mu \mathrm{L}\right)$ & $1.07(1.03-1.10)$ & $<.0001$ \\
eGFR (ml/min/1.73m²) & $1.00(0.99-1.00)$ & 0.123 \\
Serum ALT (U/dL) & $0.98(0.97-0.99)$ & $<.0001$ \\
Serum GGT (U/dL) & $1.00(1.00-1.01)$ & 0.0006 \\
Current smoking (yes; 1, no; 0) & $1.37(1.15-1.64)$ & 0.0005 \\
Serum AST (U/dL) & $1.02(1.02-1.03)$ & $<.0001$ \\
no; 0) & $1.21(1.08-1.36)$ & 0.001 \\
no; 0) & $0.87(0.77-0.99)$ & 0.031 \\
Alcohol consumption & $0.91(0.84-0.97)$ & 0.007 \\
\hline
\end{tabular}

\section{Discussion}

In the present study, we have used auto-AI and conventional analyses to show that a high AST/ALT ratio is more closely associated than low serum ALT activity with all-cause mortality in older community-dwelling Japanese people. The AI showed that older age and male sex, which are unmodifiable factors, are the first and the second greatest contributors to the risk of mortality (Table 2), as expected. However, in addition, of the biochemical parameters that are conventionally measured in clinical practice, high AST/ALT ratio was the greatest contributor to the risk of mortality, more so than each individual component; and serum albumin concentration, white blood cell count, and BMI, all of which are well-known risk factors for mortality, also contribute [36,37]. These findings were generated in a single $\mathrm{AI}$ analysis, which is a novel approach, but are consistent with previous findings of an association between AST/ALT ratio and high mortality $[7,8,10]$.

As also shown in previous studies [1-6,9,11,12], we found that people with low serum ALT activity are at a higher risk of mortality. However, the ranges of serum ALT activity that made positive and negative contributions to mortality overlapped (Figure 1 and Figure 3), which may have reduced the overall contribution calculated for serum ALT activity in the present study. However, the contribution of serum AST to mortality was lower (the tenth greatest contributor) than those of other parameters, including serum ALT activity alone (seventh greatest contributor) in the present AI analysis (Table 2). Although many studies have shown associations between low serum ALT activity and mortality [1$6,9,11,12]$, few have shown that high serum AST activity is a risk factor for mortality $[18,19]$. Therefore, the present findings are consistent with those of the majority of the previous studies concerning serum ALT and AST. Thus, the AST/ALT ratio may be a better predictor of all-cause mortality than the ALT and AST activities alone, and this was confirmed in the present study using both auto-AI and conventional statistical analyses. 
Frailty and sarcopenia are strongly associated with multiple adverse clinical outcomes, including death [38-40]. However, the identification of these conditions, and particularly of frailty, is time-consuming and labor-intensive, compared with the use of blood testing [4143]. In addition, a consensus regarding the definitions of frailty and sarcopenia has not yet been reached $[40,44]$. Therefore, it is possible that the identification of a useful circulating biomarker may help identify older people who are at a high risk of death in the near future.

The normal ranges of serum aminotransferase activities are controversial $[45,46]$. Therefore, preferably, not only the upper but also the lower limits of normal should be determined for each enzyme in each population. Otherwise, the interpretations of similar results will continue to differ between countries and investigators, leading to conflicting conclusions. In addition, age at least should be considered in the evaluation of serum aminotransferase activities because serum ALT activity likely decreases and serum AST likely increases with age [47-49].

In conventional survival analyses, the weightings of the contributions of each parameter to the outcome are not shown (Table 3), whereas the levels of both positive and negative contributions are indicated until third degrees in the auto-AI system used in the present analysis, which represents its most significant advantage over conventional statistical analyses. Therefore, at present, simultaneous analyses using conventional and AIbased methods may be superior to the use of either alone to confirm and interpret data, and to facilitate the most appropriate investigation of the underlying mechanisms, even if the results of each differ somewhat.

The present study had some limitations. First, the individual causes of death of the participants were not available. Different results may have been obtained if specific causes of death had been considered. Second, the time elapsed until death was not considered in the AI analysis because the AUC became very high (92\%) when the factor of "time elapsed until death" was included in the predictive model; thus, we excluded this factor from the list of potential contributing factors. Third, we only used a single auto-AI, Prediction One, which is now available in Japan [33], with some limited conditions. Therefore, other AIs and different conditions, such as with respect to cross-validation and the adjustment for unbalanced data, may have yielded different results. Larger studies that include an AI analysis are thus needed to confirm the present findings.

\section{Conclusions}

In the present study, we used a combination of AI and conventional statistical analysis to show that from among the standard biochemical parameters, high AST/ALT ratio may be best associated with all-cause mortality in older people, following the contributions of age and sex.

Author Contributions: K.N. and T.N. designed the study. M.Y. and K.T. acquired data from Yamato City. T.N. and K.T. established the contract between Yamato City and Kanagawa University of Human Services. K.N. and M.Y. performed the initial analysis and contributed to discussion. K.N. prepared the first draft of the manuscript. All the authors read and edited the manuscript. All the authors have read and agreed to the published version of the manuscript.

Funding: This research received no external funding.

Institutional Review Board Statement: The study was conducted according to the principles of the Declaration of Helsinki and approved by the Institutional Review Board of the Ethics Committee of Kanagawa University of Human Services (17-26).

Acknowledgments: We thank Dr. Masahito Katsuki, Neurosurgery, Itoigawa General Hospital, Itoigawa, Japan for helpful suggestions about the AI. We thank Mark Cleasby, PhD from Edanz (https://jp.edanz.com/ac) for editing a draft of this manuscript.

\section{References}

1. Elinav E, Ackerman Z, Maaravi Y, Ben-Dov IZ, Ein-Mor E, Stessman J. Low alanine aminotransferase activity in older people is 
associated with greater long-term mortality. J Am Geriatr Soc. 2006 Nov;54(11):1719-24.

2. Ruhl CE, Everhart JE. The association of low serum alanine aminotransferase activity with mortality in the US population. Am J Epidemiol. 2013 Dec 15;178(12):1702-11.

3. Liu Z, Ning H, Que S, Wang L, Qin X, Peng T. Complex association between alanine aminotransferase activity and mortality in general population: a systematic review and meta-analysis of prospective studies. PLoS One. 2014 Mar 14;9(3):e91410.

4. Oh CM, Won YJ, Cho H, Lee JK, Park BY, Jun JK, Koh DH, Ki M, Jung KW, Oh IH. Alanine aminotransferase and gammaglutamyl transferase have different dose-response relationships with risk of mortality by age. Liver Int. 2016 Jan;36(1):126-35.

5. Deetman PE, Alkhalaf A, Landman GW, Groenier KH, Kootstra-Ros JE, Navis G, Bilo HJ, Kleefstra N, Bakker SJ. Alanine aminotransferase and mortality in patients with type 2 diabetes (ZODIAC-38). Eur J Clin Invest. 2015 Aug;45(8):807-14.

6. Karaphillis E, Goldstein R, Murphy S, Qayyum R. Serum alanine aminotransferase levels and all-cause mortality. Eur J Gastroenterol Hepatol. 2017 Mar;29(3):284-288.

7. Zoppini G, Cacciatori V, Negri C, Stoico V, Lippi G, Targher G, Bonora E. The aspartate aminotransferase-to-alanine aminotransferase ratio predicts all-cause and cardiovascular mortality in patients with type 2 diabetes. Medicine (Baltimore). 2016 Oct;95(43):e4821.

8. Yokoyama M, Watanabe T, Otaki Y, Takahashi H, Arimoto T, Shishido T, Miyamoto T, Konta T, Shibata Y, Daimon M, Ueno Y, Kato T, Kayama T, Kubota I. Association of the Aspartate Aminotransferase to Alanine Aminotransferase Ratio with BNP Level and Cardiovascular Mortality in the General Population: The Yamagata Study 10-Year Follow-Up. Dis Markers. 2016;2016:4857917.

9. Schmilovitz-Weiss H, Gingold-Belfer R, Boltin D, Beloosesky Y, Meyerovitch J, Tor R, Issa N, Grossman A, Koren-Morag N, Weiss A. Risk of mortality and level of serum alanine aminotransferase among community-dwelling elderly in Israel. Eur J Gastroenterol Hepatol. 2018 Dec;30(12):1428-1433.

10. Feng X, Wen Y, Peng FF, Wang N, Zhan X, Wu X. Association between aminotransferase/alanine aminotransferase ratio and cardiovascular disease mortality in patients on peritoneal dialysis: a multi-center retrospective study. BMC Nephrol. 2020 Jun $1 ; 21(1): 209$

11. Visaria A, Pai S, Fayngersh A, Kothari N. Association between alanine aminotransferase within the normal range and all-cause and cause-specific mortality: A nationwide cohort study. PLoS One. 2020 Nov 20;15(11):e0242431.

12. An SJ, Yang YJ, Jeon NM, Hong YP, Kim YI, Kim DY. Significantly Reduced Alanine Aminotransferase Level Increases AllCause Mortality Rate in the Elderly after Ischemic Stroke. Int J Environ Res Public Health. 2021 May 5;18(9):4915. 
13. Maeda D, Kagiyama N, Jujo K, Saito K, Kamiya K, Saito H, Ogasahara Y, Maekawa E, Konishi M, Kitai T, Iwata K, Wada H, Hiki M, Dotare T, Sunayama T, Kasai T, Nagamatsu H, Ozawa T, Izawa K, Yamamoto S, Aizawa N, Yonezawa R, Oka K, Momomura SI, Matsue Y. Aspartate aminotransferase to alanine aminotransferase ratio is associated with frailty and mortality in older patients with heart failure. Sci Rep. 2021 Jun 7;11(1):11957.

14. Nakamura K, Okamura T, Kanda H, Hayakawa T, Okayama A, Ueshima H; Health Promotion Research Committee of the Shiga National Health Insurance Organizations. The value of combining serum alanine aminotransferase levels and body mass index to predict mortality and medical costs: a 10-year follow-up study of National Health Insurance in Shiga, Japan. J Epidemiol. 2006 Jan;16(1):15-20.

15. Yun KE, Shin CY, Yoon YS, Park HS. Elevated alanine aminotransferase levels predict mortality from cardiovascular disease and diabetes in Koreans. Atherosclerosis. 2009 Aug;205(2):533-7.

16. Shim JJ, Kim JW, Oh CH, Lee YR, Lee JS, Park SY, Kim BH, Oh IH. Serum alanine aminotransferase level and liver-related mortality in patients with chronic hepatitis B: A large national cohort study. Liver Int. 2018 Oct;38(10):1751-1759.

17. Li J, Zhao Z, Jiang H, Jiang M, Yu G, Li X. Predictive value of elevated alanine aminotransferase for in-hospital mortality in patients with acute myocardial infarction. BMC Cardiovasc Disord. 2021 Feb 9;21(1):82.

18. 18 Ravel V, Streja E, Molnar MZ, Rezakhani S, Soohoo M, Kovesdy CP, Kalantar-Zadeh K, Moradi H. Association of aspartate aminotransferase with mortality in hemodialysis patients. Nephrol Dial Transplant. 2016 May;31(5):814-22.

19. Xie K, Chen CH, Tsai SP, Lu PJ, Wu H, Zeng Y, Ye Y, Tu H, Wen C, Huang M, Zhang Y, Lee JH, Tsai MK, Wen CP, Wu X. Loss of Life Expectancy by 10 Years or More From Elevated Aspartate Aminotransferase: Finding Aspartate Aminotransferase a Better Mortality Predictor for All-Cause and Liver-Related than Alanine Aminotransferase. Am J Gastroenterol. 2019 Sep;114(9):1478-1487.

20. Shibata M, Nakajima K. High Serum Aspartate Aminotransferase, Underweight, and Weight Loss in Older People: Results of the KITCHEN-4. Healthcare (Basel). 2020 Mar 25;8(2):69.

21. Panteghini, M. Aspartate aminotransferase isoenzymes. Clin Biochem. 1990, 23, 311-319.

22. Lala, V.; Minter, D.A. Liver Function Tests; Stat Pearls Publishing: Treasure Island, FL, USA, 2019.

23. Nathwani, R.A.; Pais, S.; Reynolds, T.B.; Kaplowitz, N. Serum alanine aminotransferase in skeletal muscle diseases. Hepatology $2005,41,380-382$.

24. Malakouti, M.; Kataria, A.; Ali, S.K.; Schenker, S. Elevated Liver Enzymes in Asymptomatic Patients - What Should I Do? J. Clin. Transl. Hepatol. 2017, 5, 394-403. 
25. Tomizawa M, Kawanabe Y, Shinozaki F, Sato S, Motoyoshi Y, Sugiyama T, Yamamoto S, Sueishi M. Elevated levels of alanine transaminase and triglycerides within normal limits are associated with fatty liver. Exp Ther Med. 2014 Sep;8(3):759-762.

26. Martin-Rodriguez JL, Gonzalez-Cantero J, Gonzalez-Cantero A, Arrebola JP, Gonzalez-Calvin JL. Diagnostic accuracy of serum alanine aminotransferase as biomarker for nonalcoholic fatty liver disease and insulin resistance in healthy subjects, using 3T MR spectroscopy. Medicine (Baltimore). 2017 Apr;96(17):e6770.

27. Liu Z, Que S, Xu J, Peng T. Alanine aminotransferase-old biomarker and new concept: a review. Int J Med Sci. 2014 Jun 26;11(9):925-35.

28. Choi YJ, Kwon DS, Kim T, Cho JH, Kim HJ, Byun MK, Park HJ. Low alanine aminotransferase as a risk factor for chronic obstructive pulmonary disease in males. Sci Rep. 2021 Jul 21;11(1):14829.

29. Romiti S, Vinciguerra M, Saade W, Anso Cortajarena I, Greco E. Artificial Intelligence (AI) and Cardiovascular Diseases: An Unexpected Alliance. Cardiol Res Pract. 2020 Jun 27;2020:4972346.

30. https://www.kuhs.ac.jp/research/news/details 00248.html

31. http://www.city.yamato.lg.jp/web/kouhou/n20181218-2.html

32. Matsuo S, Imai E, Horio M, Yasuda Y, Tomita K, Nitta K, Yamagata K, Tomino Y, Yokoyama H, Hishida A; Collaborators developing the Japanese equation for estimated GFR. Revised equations for estimated GFR from serum creatinine in Japan. Am J Kidney Dis. 2009 Jun;53(6):982-92.

33. Sony Network Communications, Prediction One; 2020. Available from: https://www.predictionone.sony.biz. Last accessed on July 29, 2021.

34. Katsuki M, Kawamura S, Koh A. Easily Created Prediction Model Using Automated Artificial Intelligence Framework (Prediction One, Sony Network Communications Inc., Tokyo, Japan) for Subarachnoid Hemorrhage Outcomes Treated by Coiling and Delayed Cerebral Ischemia. Cureus. 2021 Jun 16;13(6):e15695.

35. https://towardsdatascience.com/be-careful-when-interpreting-your-features-importance-in-xgboost-6e16132588e7

36. Agarwal N, Acevedo F, Leighton LS, Cayten CG, Pitchumoni CS. Predictive ability of various nutritional variables for mortality in elderly people. Am J Clin Nutr. 1988 Nov;48(5):1173-8.

37. Hirani V, Naganathan V, Blyth F, Le Couteur DG, Gnjidic D, Stanaway FF, Seibel MJ, Waite LM, Handelsman DJ, Cumming RG. Multiple, but not traditional risk factors predict mortality in older people: the Concord Health and Ageing in Men Project. Age (Dordr). 2014;36(6):9732.

38. Landi F, Cruz-Jentoft AJ, Liperoti R, Russo A, Giovannini S, Tosato M, Capoluongo E, Bernabei R, Onder G. Sarcopenia and 
mortality risk in frail older persons aged 80 years and older: results from ilSIRENTE study. Age Ageing. 2013 Mar;42(2):203-9.

39. Chang SF, Lin PL. Systematic Literature Review and Meta-Analysis of the Association of Sarcopenia With Mortality. Worldviews Evid Based Nurs. 2016 Apr;13(2):153-62.

40. Landi F, Calvani R, Cesari M, Tosato M, Martone AM, Ortolani E, Savera G, Salini S, Sisto A, Picca A, Marzetti E. Sarcopenia: An Overview on Current Definitions, Diagnosis and Treatment. Curr Protein Pept Sci. 2018 May 14;19(7):633-638.

41. Rustani K, Kundisova L, Capecchi PL, Nante N, Bicchi M. Ultrasound measurement of rectus femoris muscle thickness as a quick screening test for sarcopenia assessment. Arch Gerontol Geriatr. 2019 Jul-Aug;83:151-154.

42. Berg HE, Truong D, Skoglund E, Gustafsson T, Lundberg TR. Threshold-automated CT measurements of muscle size and radiological attenuation in multiple lower-extremity muscles of older individuals. Clin Physiol Funct Imaging. 2020 May;40(3):165172.

43. Meerkerk CDA, Chargi N, de Jong PA, van den Bos F, de Bree R. Low skeletal muscle mass predicts frailty in elderly head and neck cancer patients. Eur Arch Otorhinolaryngol. 2022 Feb;279(2):967-977.

44. Cederholm T. Overlaps between Frailty and Sarcopenia Definitions. Nestle Nutr Inst Workshop Ser. 2015;83:65-9.

45. Wu WC, Wu CY, Wang YJ, Hung HH, Yang HI, Kao WY, Su CW, Wu JC, Chan WL, Lin HC, Lee FY, Lee SD. Updated thresholds for serum alanine aminotransferase level in a large-scale population study composed of 34346 subjects. Aliment Pharmacol Ther. 2012 Sep;36(6):560-8.

46. Kolahdoozan S, Mirminachi B, Sepanlou SG, Malekzadeh R, Merat S, Poustchi H. Upper Normal Limits of Serum Alanine Aminotransferase in Healthy Population: A Systematic Review. Middle East J Dig Dis. 2020 Jul;12(3):194-205.

47. Dong MH, Bettencourt R, Barrett-Connor E, Loomba R. Alanine aminotransferase decreases with age: the Rancho Bernardo Study. PLoS One. 2010 Dec 8;5(12):e14254.

48. Vespasiani-Gentilucci U, Gallo P, Piccinocchi G, Piccinocchi R, Schena E, Galati G, De Vincentis A, Dell'Unto C, Picardi A. Determinants of alanine aminotransferase levels in a large population from Southern Italy: relationship between alanine aminotransferase and age. Dig Liver Dis. 2014 Oct;46(10):909-15.

49. Shibata M, Nakajima K, Higuchi R, Iwane T, Sugiyama M, Nakamura T. High Concentration of Serum Aspartate Aminotransferase in Older Underweight People: Results of the Kanagawa Investigation of the Total Check-Up Data from the National Database-2 (KITCHEN-2). J Clin Med. 2019 Aug 22;8(9):1282. 